\title{
Prise en charge bucco-dentaire d'une jeune patiente atteinte d'hypoplasie dermique en aires de Goltz
}

\section{RÉSUMÉ}

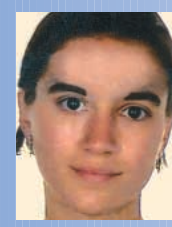

\section{Diane DOUYERE}

Assistante hospitalo-universitaire Service d'odontologie de I'hôpital Bretonneau, Paris. Faculté de chirurgie dentaire, Paris Descartes.

\section{Aurélie BESLOT-NEVEU}

Assistante hospitalo-universitaire, Service d'odontologie de I'hôpital Albert Chenevier, Créteil. Faculté de chirurgie dentaire, Paris Descartes.

\section{Laurent PIERRISNARD}

Professeur des universités, Praticien hospitalier, Service d'odontologie de l'hôpital Bretonneau, Paris. Faculté de chirurgie dentaire, Paris Descartes.

\section{Françoise VILLETTE}

Maître de conférence des universités, Praticien hospitalier, Service d'odontologie de l'hôpital Bretonneau, Paris. Faculté de chirurgie dentaire, Paris Descartes.
L'hypoplasie dermique en aires de Goltz est un syndrome génétique polydysplasique affectant les tissus d'origines ectodermique et mésodermique. Parmi l'ensemble des signes cliniques, les troubles bucco-dentaires occupent une place importante. La prise en charge thérapeutique de ces patients est complexe tant les affections dentaires et parodontales sont variées dans leur forme et leur sévérité. Nous présentons ici le cas d'une jeune patiente suivie dans le service d'odontologie de l'Hôpital Bretonneau.
- hypoplasie dermique en aires de Goltz

affections dentaires et parodontales

- thérapeutiques bucco-dentaires 


\section{Introduction}

$>$

L'hypoplasie dermique en aires de

Goltz est un syndrome génétique polydysplasique affectant les tissus d'origine ectodermique et mésodermique. Évoquée pour la première fois par Jessner en 1921, son nom définitif ne lui a été attribué qu'en 1962 par Goltz et al. [8]. On le retrouve dans la littérature sous la dénomination de FDH (Focal Dermal Hypoplasia) pour les Anglo-Saxons, ou de syndrome de Goltz (ou de Goltz-Gorlin). Cette pathologie est référencée dans le MIM (Mendelian Inheritance in Man) sous I'appellation MIM 305600 [14].

En 2004, un peu plus de 200 cas avaient été rapportés dans le monde [3]. Cette pathologie touche majoritairement les individus de sexe féminin, avec un ratio hommes/femmes de 11/150 [10], soit moins de $10 \%$ de patients masculins.

L'étiologie de cette pathologie est encore inconnue à notre époque. Son mode de trans- mission serait vraisemblablement lié au chromosome $\mathrm{X}$ selon un mode dominant ou autosomal dominant. II existe une très fréquente létalité in utero des fœtus de sexe masculin homozygotes, expliquant le ratio vu précédemment $[3,15]$.

La mutation simple d'un gène à expressivité variable qui serait localisé en Xp22.31 serait à l'origine de ce syndrome. En effet, certains patients présentent une délétion du bras court du chromosome $X$ avec un point de rupture en ce lieu [6].

Les signes cliniques principaux sont, dans l'ordre de fréquence d'apparition, cutanés et osseux (dans $100 \%$ des cas), bucco-dentaires (dans $50 \%$ des cas [15]) et oculaires (dans $40 \%$ des cas [3]). Des atteintes thoraco-abdominales, cardiaques, rénales et urogénitales sont souvent observées.

\section{Signes cliniques généraux}

Les structures cutanées sont touchées chez l'ensemble des patients présentant ce syndrome (fig. 1 a et b). La peau est atrophiée, fine, sèche et desquamée et présente des aires linéaires d'hypo- ou d'hyperpigmentation. Une biopsie à ce niveau révélerait une atrophie de l'épiderme associée à une hernie du tissu adipeux $[5,13]$. Des lésions cutanées de différents types peuvent être relevées, à des fréquences variables : télangiectasies, nodules ou macules, papillomes, lésions verruqueuses, bourgeons, kystes ou tumeurs cutanées [15]. L'ensemble de ces troubles cutanés entraîne pour le patient de sévères gênes fonctionnelles, esthétiques, et nécessite un traitement lourd et contraignant au quotidien, le plus souvent par l'application très régulière de crèmes dermatologiques.

Les structures osseuses sont concernées et des anomalies squelettiques seront relevées, principalement au niveau des pieds et des mains : syndactylies, polydactylies (fig. 2). Elles pourront chez certains patients être source de difficultés motrices et de dextérité limitée. Les métacarpes sont de plus petite taille, voire inexistants [15].

Les os longs présentent également des déformations. La trabéculation osseuse est grossière. 

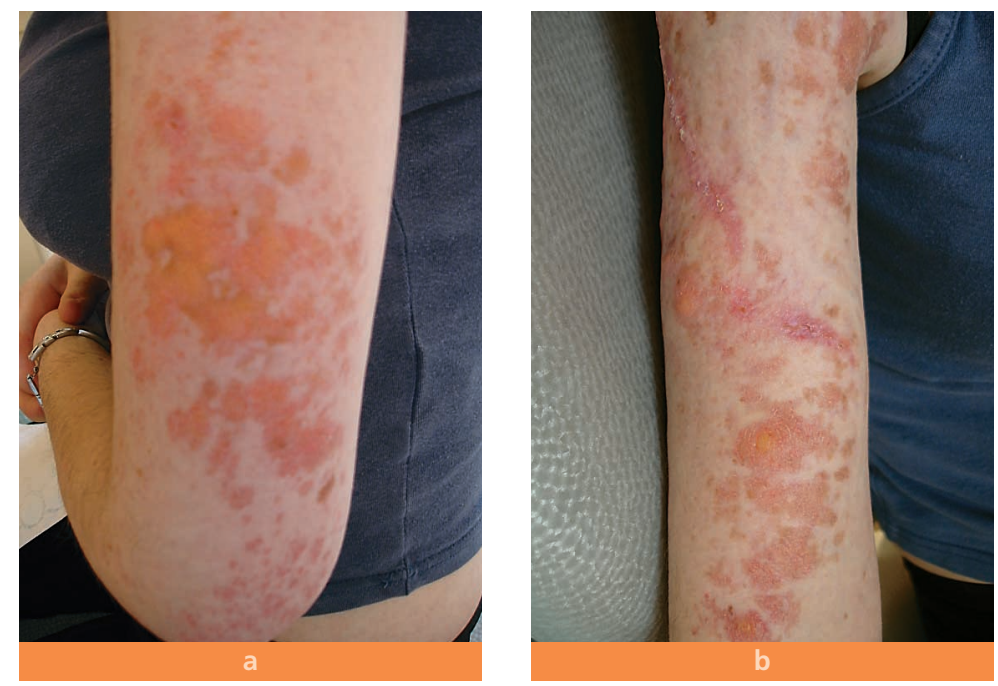

Fig. 1 a et $b$ Photographies du bras gauche et du bras droit de la patiente. La cicatrice visible sur le bras droit signe une intervention d'élimination de lésions cutanées trop invalidantes.

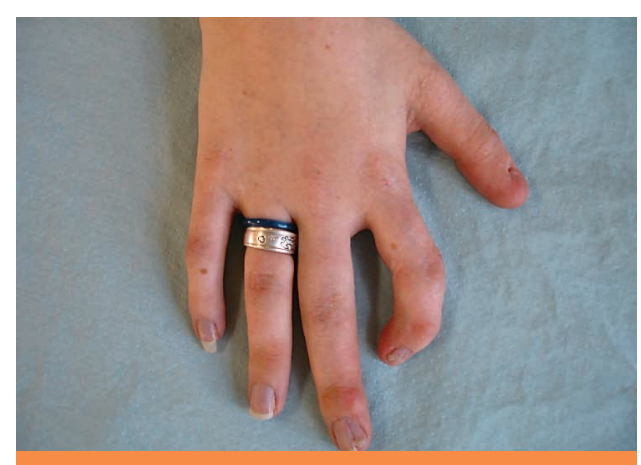

Fig. 2 Photographie de la main droite de la patiente, posée à plat sur un support dur. Noter les anomalies de disposition des métacarpes.
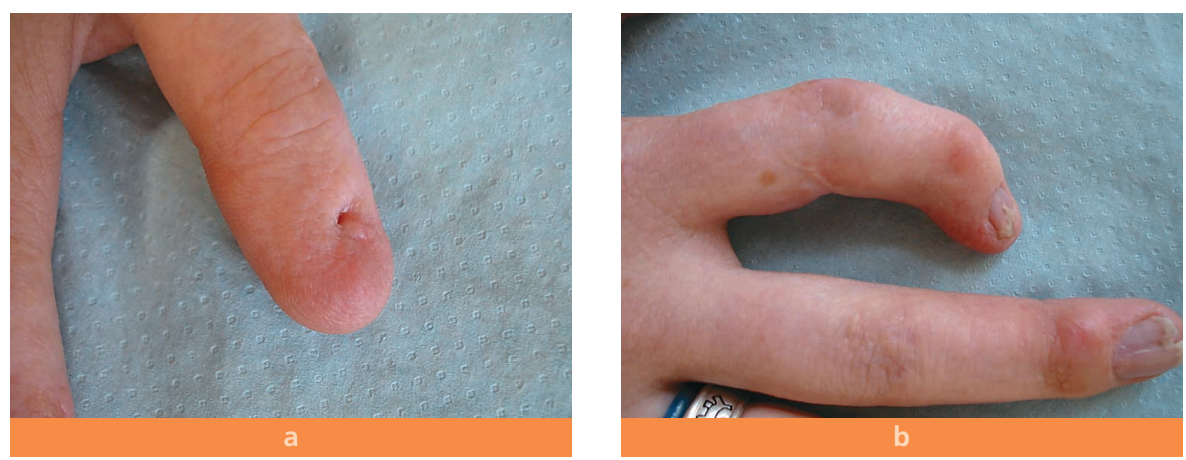

Fig. 3 a et b Photographie du pouce droit de la patiente montrant l'aplasie complète de l'ongle et photographie de l'index et du majeur droits montrant les fentes longitudinales sur les ongles. 
Les patients atteints sont de petite taille et certains présentent même un nanisme. De nombreux problèmes dorsaux se manifestent, sous forme d'anomalies des diaphyses, de spina bifida, et plus globalement de scoliose [15].

Présentes dans $40 \%$ des cas, les atteintes oculaires se traduisent le plus souvent par une microphtalmie voire une anophtalmie, et un colobome de l'iris ou de la rétine, associé ou non à des défauts congénitaux [15]. Sont également fréquemment retrouvés : un strabisme, un nystagmus et une photophobie. II convient donc, pour le chirurgien-dentiste, de modérer I'utilisation du scialytique au cabinet dentaire. Les phanères montrent également des anomalies. Les sourcils et les cheveux sont fins et clair- semés (hypotrichie), voire absents (atrichie). Les ongles sont hypoplasiques voire aplasiques [15]. Ils présentent des fentes longitudinales [13] (fig. 3 a et b).

L'oreille est également cible d'anomalies. On relève des troubles de l'oreille moyenne, des troubles anatomiques (oreille proéminente et/ou décollée), et des diminutions du champ d'audition pouvant aller jusqu'à la surdité.

Enfin, certaines atteintes plus occasionnelles touchent d'autres organes du corps.

Un retard mental et/ou une hydrocéphalie peuvent exister. Les troubles cardiaques, rénaux, et urogénitaux, plus rares et très variables, s'ils apparaissent, déterminent en général l'espérance de vie de ces patients [1].

\section{Signes bucco-dentaires}

La fréquence des signes cliniques place les signes bucco-dentaires en troisième position. Ces patients présentent une macrostomie. Les bases osseuses, maxillaire et mandibulaire, sont hypodéveloppées (fig. 4). La voûte palatine est haute et creuse [2].

La partie centrale de la face, les sinus maxillaires et les narines sont hypodéveloppés.

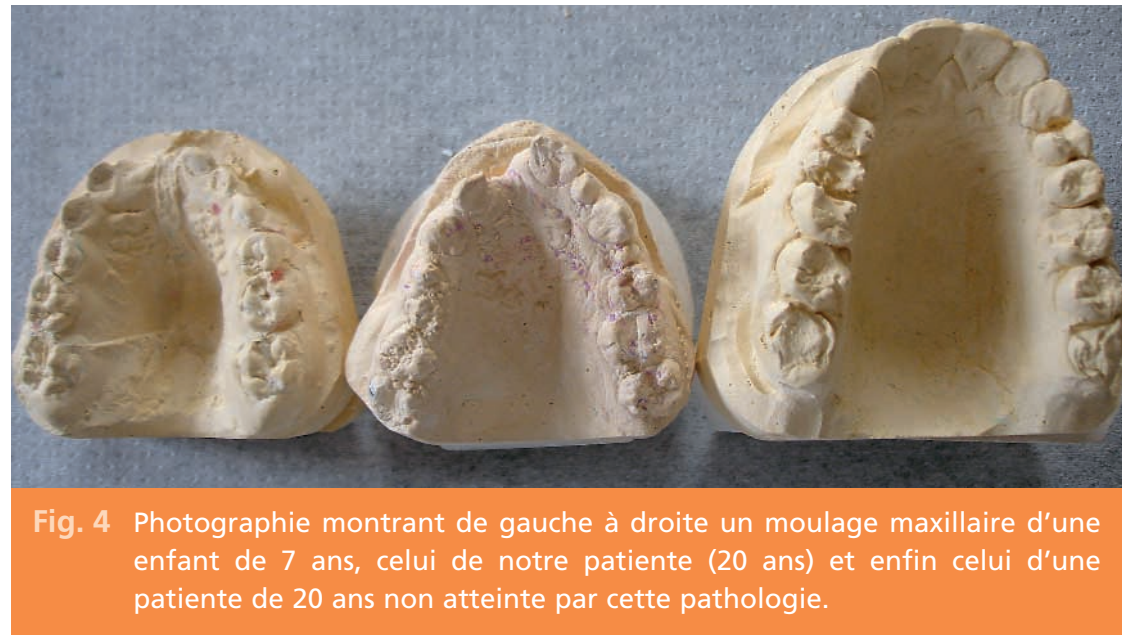

Le visage est asymétrique. II n'existe pas de paralysie faciale associée. On constate un prognathisme mandibulaire donnant un aspect de menton pointu [2] (fig. 5).

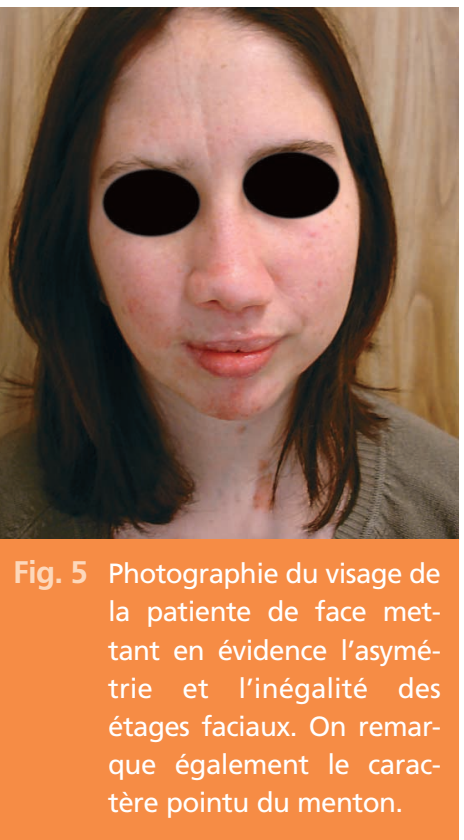


Concernant les structures gingivales et muqueuses, on note fréquemment la présence de fentes labiale latérale ou palatine complète. Les freins sont à insertion haute et multiple. Le signe parodontal le plus fréquemment observé est une dysplasie gingivale, caractérisée par une gencive très fine, inflammatoire et parfois hémorragique [14]. Une gingivite marginale se développe en regard de l'ensemble des dents. De nombreux papillomes se développent sur les lèvres (jonction cutanéo- muqueuse), ainsi que sur la langue et les gencives [15] (fig. 6).

Les organes dentaires sont touchés à différents niveaux et de multiples conséquences peuvent être observées: dystrophie dentaire, microdontie, dents pointues et/ou coniques, et plus rarement un taurodontisme et des résorptions radiculaires externes [4]. Les phénomènes de dentition sont altérés (fig. 7 a et b et fig. 8 a et b). II en résulte des hypodonties, des oligodonties, des éruptions ecto-
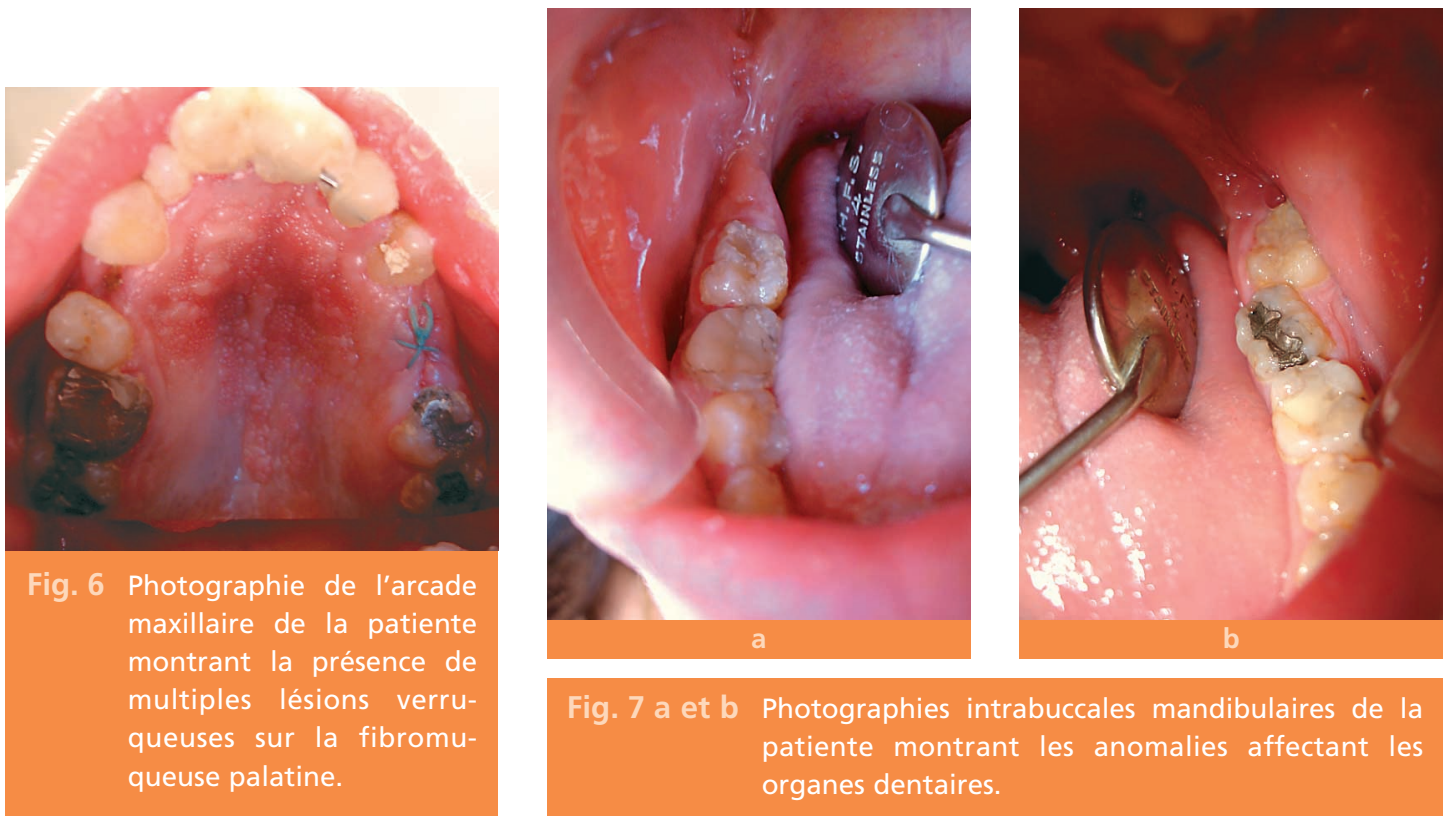

Fig. 7 a et b Photographies intrabuccales mandibulaires de la patiente montrant les anomalies affectant les organes dentaires.
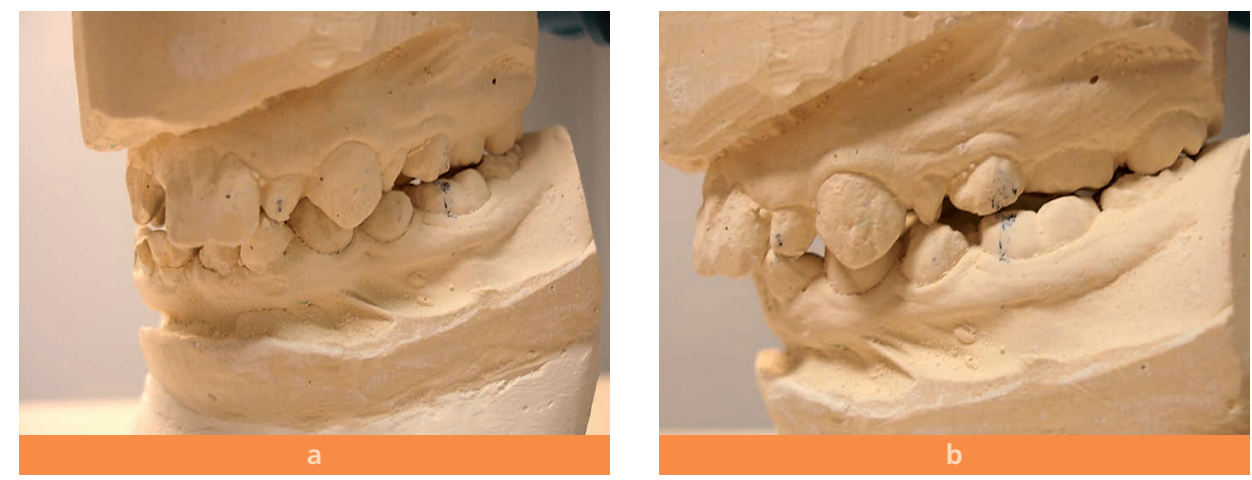

Fig. 8 a et b Moulages de la patiente montés en articulateur et mettant en évidence l'ensemble des troubles de la dentition. 
piques et/ou retardées, des malocclusions, des germes surnuméraires, et plus occasionnellement des fusions et des géminations des dents temporaires [15]. Histologiquement, I'émail

\section{La lyonisation $[3,15]$}

Parmi I'ensemble de ces signes cliniques, certains s'expliquent par le phénomène de lyonisation, phénomène encore en cours d'étude aujourd'hui.

Pendant la phase de différenciation cellulaire, le chromosome $X$ est inactivé dans chaque cellule somatique par «le centre d'inactivation du X» localisé en Xq13. À partir de ce chromosome inactivé est transcrit le XIST ( $X$ Inactivation Specific Transcript), dont la propagation au fil des multiplications cellulaires est perma- est hypoplasique, ce qui explique en partie l'apparition de caries précoces. L'ensemble de ces anomalies s'exprime de façon variable d'un patient à l'autre.

nente et clonale, selon la loi du hasard. II en résulte une alternance de clones cellulaires à phénotype sain et de clones cellulaires à phénotype altéré.

Les améloblastes, les kératinocytes et les ostéoblastes subissent ce phénomène de lyonisation et cela se traduit cliniquement et respectivement par : une hypoplasie amélaire, des lignes cutanées de Blaschko et des stries linéaires parallèles au grand axe des os longs, observables radiologiquement.

\section{Prise en charge bucco-dentaire}

Mlle $\mathrm{H}, 20$ ans, se présente à la consultation avec une demande principalement d'ordre esthétique, pour, selon ses dires, réduire la proéminence de ses dents antérieures maxillaires (fig. 9 a à c). Le traitement doit être rapide, compte tenu des faibles disponibilités futures de la patiente.

Elle est porteuse d'une hypoplasie dermique en aires de Goltz diagnostiquée à sa naissance. Son père en est également atteint. L'entretien médical ne révèle aucune autre particularité.

Les antécédents bucco-dentaires font état de nombreux soins conservateurs (fig. 6, fig. 7 a et $\mathbf{b}$ et fig. 10), réalisés dans un cabinet, de façon ponctuelle et irrégulière. L'hypoplasie amélaire a favorisé le développement précoce de caries chez cette patiente. L'agénésie de l'incisive centrale maxillaire droite est jusqu'à présent solutionnée par une attelle provisoire maintenant une dent résine en place de 11 (fig. 6 et fig. 9 a à c). La patiente présente également une agénésie de la dent 31.

Les dents 14, 15, 24 et 32 ont été extraites dans le cadre du plan de traitement, car elles étaient non fonctionnelles, en microdontie. Pour les mêmes raisons, les dents 22 et 42 seront extraites, ainsi que la dent 26 , non conservable et à l'origine d'une cellulite.

Compte tenu de son jeune âge, aucune prise en charge globale prothétique ne lui a jamais été proposée. Elle vient consulter dans le service d'odontologie de I'hôpital Bretonneau avec l'espoir d'être réhabilitée esthétiquement et fonctionnellement. 

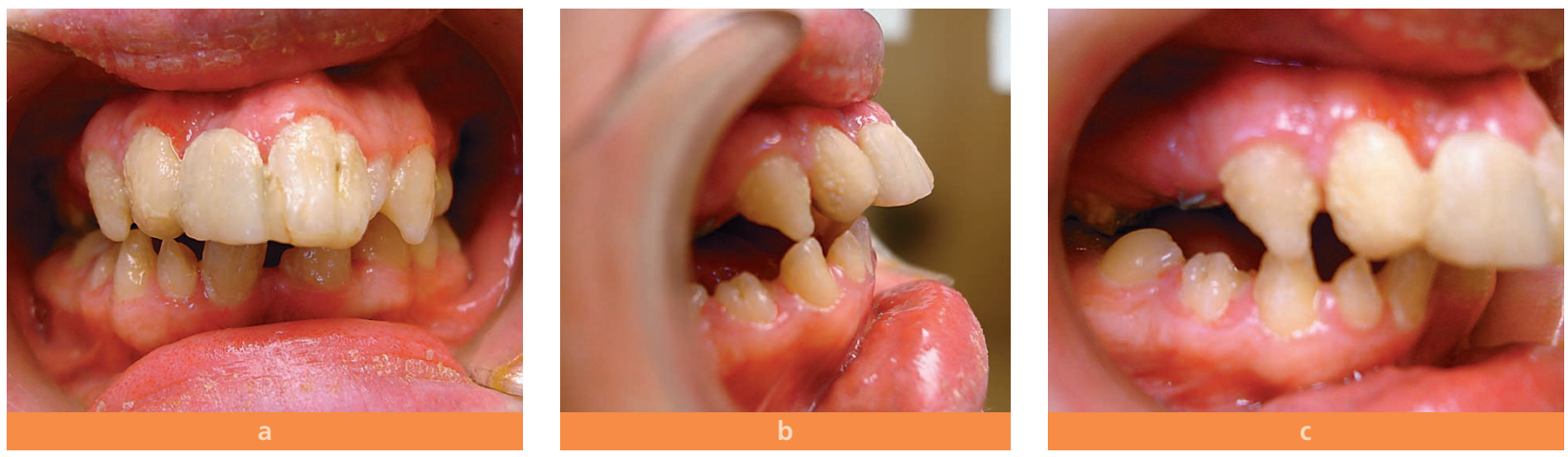

Fig. 9 a à c Photographies intrabuccales faisant état de la proalvéolie du secteur antérieur maxillaire et de la rétromandibulie.

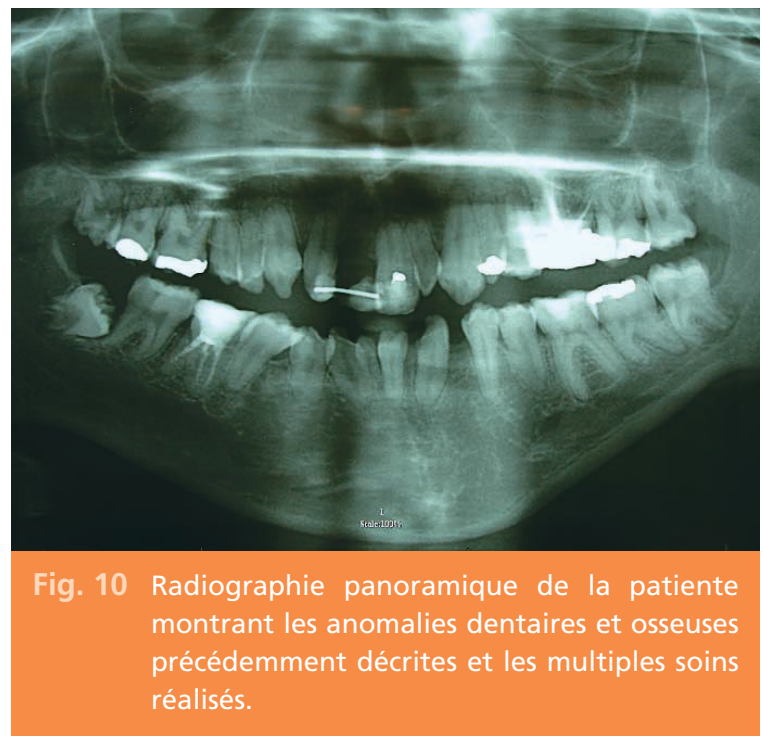

L'hypoplasie dermique en aires de Goltz a de nombreuses conséquences au niveau buccodentaire. Cette pathologie a donc eu une influence considérable dans la prise en charge thérapeutique de Mlle $\mathrm{H}$.

Au niveau parodontal, l'affection des tissus de soutien osseux, ligamentaires, cémentaires et gingivaux (gingivite chronique), entretient de nombreuses mobilités dentaires, principalement dans les secteurs antérieurs. Dans la prise en charge thérapeutique, ces problèmes parodontaux obligent à renforcer les conseils d'hygiène et à assurer un assainissement régulier.
Les actes doivent être atraumatiques compte tenu de la fragilité gingivale (pose de la digue avec précaution, adaptation rigoureuse des couronnes provisoires...).

Les anomalies des organes dentaires ont compliqué les traitements endodontiques, du fait de canaux très fins, voire calcifiés, ou au contraire d'apex très larges comme sur la dent 21, mais également les réalisations prothétiques du fait d'axes dentaires multiples et variés, de rétention non optimale, rendant l'intégration esthétique et fonctionnelle des prothèses plus difficile à obtenir. 
De nombreuses contraintes nous ont donc été imposées et les objectifs de traitement posés sont :

- une prise en charge parodontale et un suivi cariologique réguliers afin d'intercepter les phases d'exacerbation de la pathologie pendant lesquelles les signes cliniques s'aggravent (hyperplasie gingivale et gingivite marginale sévères) ;

- l'élimination des foyers infectieux (26) et des dents non fonctionnelles (microdonties: 14, $15,22,25,32,42)$;

- la restauration des édentements tout en répondant à la demande esthétique et fonctionnelle de la patiente.

\section{Décision thérapeutique}

Plusieurs solutions thérapeutiques s'offrent pour la restauration des édentements de 11 , $14,15,22,25,26,32$ et 42.

La réalisation d'implants a été écartée du fait de l'incertitude concernant l'obtention d'une bonne ostéo-intégration et d'une bonne qualité d'ancrage compte tenu de l'influence de la pathologie sur les tissus de soutien parodontaux, et de la nécessité de réaliser un traitement dans un court laps de temps. Pour les mêmes raisons, l'apport d'un traitement orthodontique n'a pas été retenu. La réhabilitation par une prothèse amovible partielle a été écartée, cette solution ne répondant pas à l'ensemble des demandes de la patiente.

Il a donc été décidé avec l'ensemble de l'équipe soignante, la patiente et ses parents, d'entreprendre la réhabilitation bucco-dentaire de Mlle $\mathrm{H}$ par prothèse fixée avec la réalisation de trois bridges céramo-métalliques de quatre éléments au maxillaire : piliers 16-13 avec intermédiaires 14-15, piliers 12-21 avec intermédiaires $11-22$ et piliers $25-27$ avec intermédiaire 24-26. Cette solution présente le meilleur rapport coût/bénéfice/sécurité.

II a été discuté avec la patiente de l'intérêt de combler l'édentement du secteur antérieur mandibulaire conjointement à la réhabilitation prothétique maxillaire, mais Mlle $\mathrm{H}$ a refusé, sa demande étant réellement et uniquement axée sur ses dents antérieures maxillaires.
Cependant, une fois les bridges maxillaires réalisés, la patiente s'est ravisée, et a finalement accepté la réalisation d'un bridge céramo-céramique de cinq éléments restaurant l'édentement de 31, 32 et 42 .

À l'arcade maxillaire, toutes les dents piliers sont traitées endodontiquement (fig. 11 a à d). À l'arcade mandibulaire, les dents sont conservées vitales.

Certaines difficultés et particularités ont été rencontrées lors des traitements endodontiques:

- la fragilité gingivale à prendre en compte pour l'isolation sous digue ;

- la difficulté d'accès au réseau canalaire par la présence de l'attelle remplaçant la dent 11 ;

- la nécrose de la dent 21 ;

- des canaux courts et larges (secteur antérieur) ou très étroits (la dent 16) ;

- des apex larges (21) ou calcifiés (12).

Les restaurations provisoires sont réalisées. Pour ne pas compromettre l'hygiène et l'état parodontal déjà affecté par la pathologie, les éléments provisoires sont unitaires et ne concernent que les dents piliers. L'exception est faite pour le secteur antérieur maxillaire où l'attelle provisoire est remplacée par un bridge provisoire ayant pour piliers 13,12 et 21 , et restaurant 11 et 22 (fig. 12 a et b). Ce dernier est réalisé à l'aide de facettes du commerce adap- 

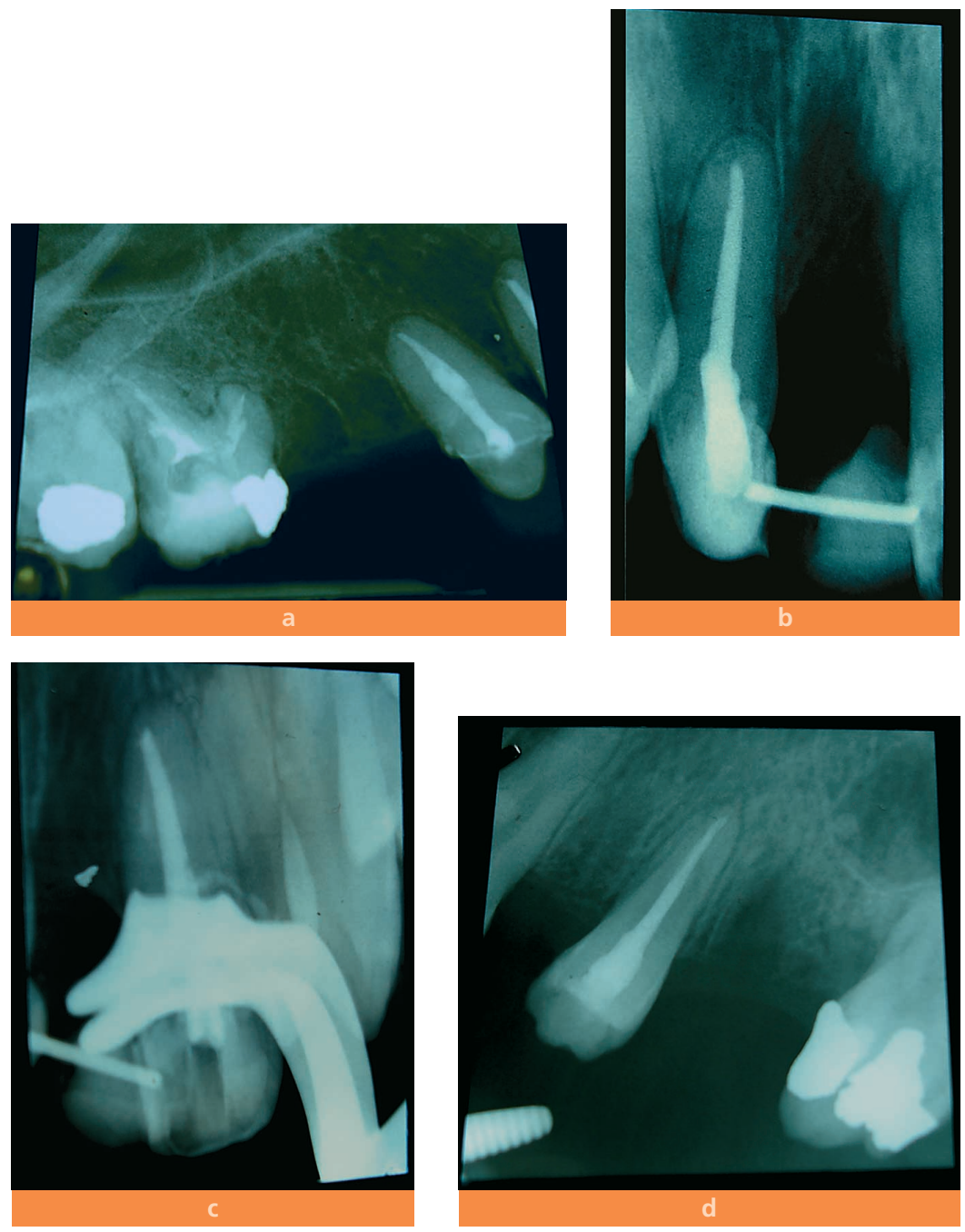

Fig. $11 \mathrm{a}$ à $\mathrm{d}$ De gauche à droite, radiographies rétroalvéolaires des traitements endodontiques de 16 et $13,12,21,25$.
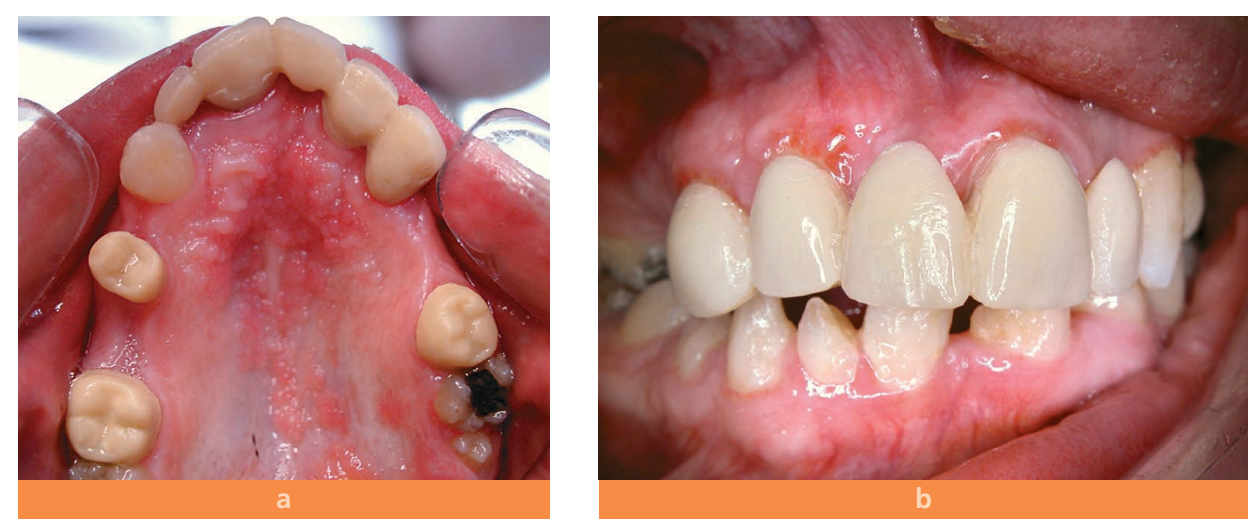

Fig. 12 a et b Photographies intrabuccales des éléments provisoires maxillaires. 

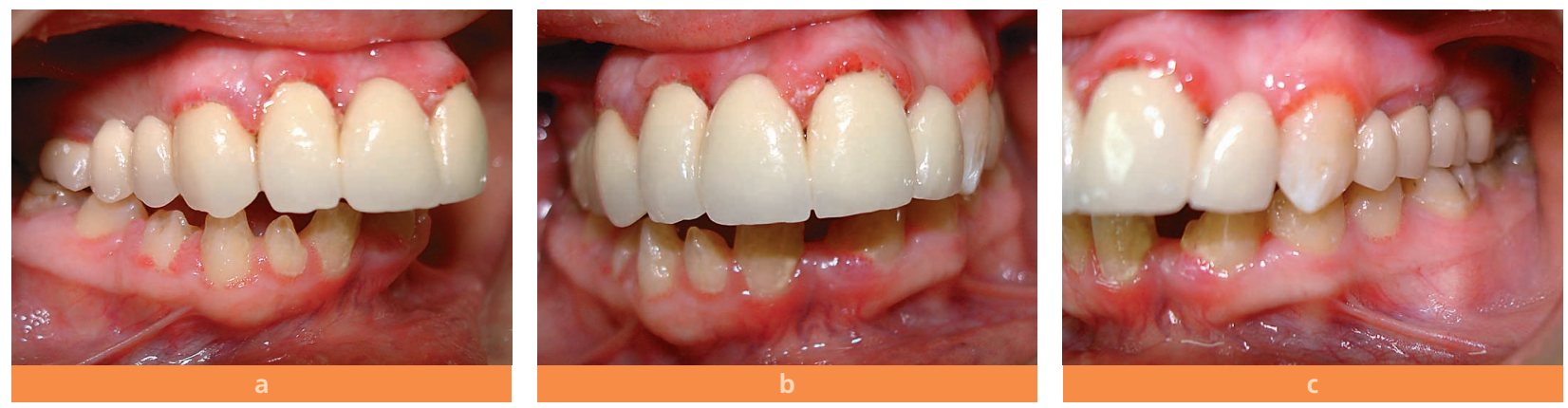

Fig. 13 a à c Photographies intrabuccales de la réhabilitation prothétique maxillaire. Noter la présence d'une gingivite marginale marquée.

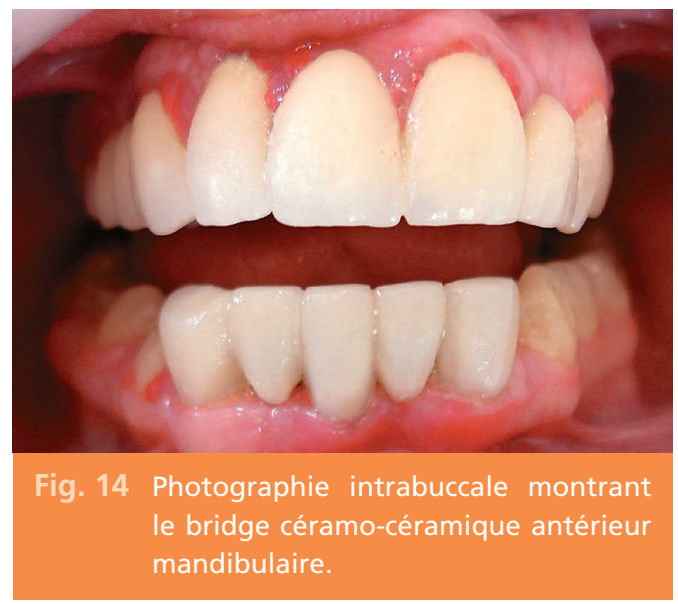

tées sur un modèle de travail puis solidarisées et amenées en bouche à l'aide d'une clé en plâtre. La partie linguale est ensuite confectionnée par apport de résine en bouche.

Des inlays cores sont réalisés sur l'ensemble des dents piliers, compte tenu :

- de l'insuffisance de la hauteur des couronnes cliniques ;

- du volume considérable des pertes de substances ;

- du manque de rétention ;

- de la nécessité de corriger les vestibulo-versions des dents du secteur incisivo-canin maxillaire ;

- de la réalisation de restaurations plurales ;

- de la nécessité d'un contrôle de l'axe d'insertion des bridges.
Sont ensuite confectionnés les trois bridges céramo-métalliques maxillaires dans un premier temps (fig. 13 a à c). L'occlusion dans laquelle s'inscrivent les prothèses est l'occlusion de convenance de la patiente. Pour répondre à la demande principale de la patiente, l'axe des incisives maxillaires est rendu plus palatin, les éléments prothétiques céramo-métalliques suivant l'axe des inlays cores sous-jacents.

La patiente nous a recontactés quelques semaines après le rendez-vous de scellement des bridges maxillaires pour nous demander de combler l'édentement du secteur antérieur mandibulaire. Satisfaite du rendu esthétique et fonctionnel des éléments maxillaires, l'édentement mandibulaire lui paraissait alors incongru. Le bridge céramo-céramique ayant pour piliers $33,41,43$ est réalisé (fig. 14). 


\section{Conclusion}

Les objectifs de traitement dans la prise en charge de patients atteints de cette pathologie sont particulièrement complexes.

D'un point de vue général, l'encadrement est multidisciplinaire, principalement d'ordre dermatologique, orthopédique pour des traitements symptomatiques, et peut faire appel à la chirurgie plastique pour l'éviction de lésions disgracieuses. Des conseils génétiques sont délivrés concernant les éventuelles générations futures. D'un point de vue bucco-dentaire, la préven- tion doit être menée le plus précocement possible et un suivi régulier installé afin d'anticiper les phases d'exacerbation de la pathologie. Notre patiente consulte régulièrement dans le service pour des contrôles rigoureux de son hygiène bucco-dentaire, de sa santé parodontale, de son risque carieux, et de la bonne intégration dans le temps des prothèses. Ce suivi instauré permet d'assurer la continuité de thérapeutiques dont le pronostic est incertain et réservé, tant la pathologie est mal connue.

\section{Bibliographie}

1. Adornato $\mathrm{MC}$, Ferras $\mathrm{S}$, Penna KJ.

Focal dermal hypoplasia Goltz Syndrome, a case report. NY State Dent J $2001 ; 67(1): 30-32$.

2. Al-Ghamdi $\mathrm{K}$, Crawford PJ. Focal dermal hypoplasia oral and dental findings. Int J Paediatr Dent 2003;13(2):121-126.

3. Balmer R, Cameron AC, Ades L, Aldred MJ. Enamel defects and Lyonization in focal dermal hypoplasia. Oral Surg Oral Med Oral Pathol Oral Radiol Endod 2004;98(6):686-691.

4. Baxter AM, Shaw MJ, Warren K.

Dental and oral lesions in two patients with focal dermal hypoplasia
(Goltz syndrome).

Br Dent J 2000;189(10):550-553.

5. Bucci E, Lo Muzio L, Mignogna MD.

Oral and dental anomalies in Goltz syndrome. J Pedod 1989;13(2):161-168.

6. Friedman PA, Rao KW, Teplin SW, Aylsworth AS. Provisional deletion mapping of the focal dermal hypoplasia (FDH) gene to Xp22.31.

Am J Hum Genet 1988;43:A50.

7. Germain D. via www.orpha.net

8. Goltz RW, Petersen WC, Gorlin RJ et al. Focal dermal hypoplasia. Arch Dermatol 1962;86:52-61.

9. Hardman CM, Garioch JJ, Eady RA, Fry L.
Focal dermal hypoplasia:

report of a case

with cutaneous

and skeletal

manifestations.

Clin Exp Dermatol

1998;23(6):281-285.

10. McNamara T, Trotman CA, Hahessy AM, Kavanagh P. Focal dermal hypoplasia (Goltz-Gorlin) syndrome with taurodontism.

Spec Care Dentist 1996;16(1):26-28.

11. National Library of Medicine via www.nlm.nih.gov

12. Ogunbiyi AO, Adewole IO, Ogunleye O, Ogunbiyi JO, Ogunseinde $\mathrm{OO}$, Baiyeroju-Agbeja A. Focal dermal hypoplasia: a case report and review of literature. West Afr J Med 2003;22(4):346-349. Review. 
13. Sacoor MF,

Motswaledi $\mathrm{MH}$.

Three cases

of focal dermal hypoplasia

(Goltz syndrome).

Clin Exp Dermatol

2005;30(1):35-37.

14. Stephen LX, Behardien N, Beighton $\mathrm{P}$.
Focal dermal hypoplasia:

management

of complex

dental features.

$\mathrm{J}$ Clin Pediatr Dent

2001;25(4):259-261.

15. Tejani Z, Batra $P$, Mason C, Atherton D.

Focal dermal hypoplasia: oral and dental findings. $\mathrm{J}$ Clin Pediatr Dent 2005;30(1):67-72. Review.

16. Terezhalmy GT, Moore WS, Bsoul SA, Flint DJ. Focal dermal hypoplasia (Goltz- Gorlin Syndrome). Quintessence Int 2002;33(9):706-707.

\section{REMERCIEMENTS}

Laboratoire de prothèses Lefebvre, François Lefebvre et Alain Vignier.

78860 Saint-Nom-La-Bretèche.

\section{SUMMARY}

\section{Dental therapeutic approach of a young patient with focal dermal hypoplasia}

Diane DOUYERE,

Aurélie BESLOT-NEVEU,

Laurent PIERRISNARD,

Françoise VILLETTE

\section{Keywords \\ - focal dermal \\ hypoplasia \\ - dental and periodontal affections \\ - dental therapeutics}

Focal dermal hypoplasia (FDH) is a polydysplasic genetic syndrome which involves ectodermic and mesodermic tissues. Among the clinical signs, dental troubles stage an important place. The therapeutic approach for these patients is complex because of the broad range in the sort and severity of dental and periodontal affections. We present here the case of a young patient with FDH followed in the dental department of Bretonneau Hospital. 